\title{
Experimental analysis of the particle transport in the magnetized plasma column with an application to the Helicon discharge
}

\author{
I. Shesterikov, ${ }^{1, a)}$ K. Crombe, ${ }^{2,3}$ and J.-M. Noterdaeme ${ }^{1,2}$ \\ 1) Max-Planck-Institut für Plasmaphysik, D-85748 Garching, Germany \\ ${ }^{2)}$ Ghent University, Department of Applied Physics, ,B-9000 Gent, Belgium \\ ${ }^{3)}$ LPP-ERM/KMS, B-1000 Brussels, Belgium
}

(Dated: 7 May 2019)

Different transport mechanisms in a magnetized radio-frequency plasma discharge in the IShTAR $^{1}$ device are compared. The total cross-field particle transport systematically shows the best agreement with the turbulent diffusion. Also, the ion mobility dominated transport could substantially contribute to radial losses. The relative role of parallel and perpendicular losses in the overall particle losses is also compared. The total perpendicular particle losses are comparable or even larger than the parallel ones, imposing a practical limitation on achieving high density plasma by a simple multiplication in the number of helicon antennae.

PACS numbers: Valid PACS appear here

Keywords: Suggested keywords

\section{INTRODUCTION}

The key step in the development of a new plasma source is the estimation of typical plasma parameters achieved for a given absorbed power $P$, neutral gas pressure $p$ and an external magnetic field $B$. Understanding the main transport mechanisms which govern the particle losses is the key step showing routes for the design optimization. The transport in the magnetized plasma cylinder could be governed either by the mobility, diffusion (collisional or turbulent) or free-fall mechanisms depending on the ion mean free path $\lambda_{i}$, strength of the magnetic field, length $L$ and radius $R$ of the cylinder, whether the side and/or end walls are conducting or dielectric. Meanwhile, the collisional transport in magnetic field is anisotropic and is based on the difference between the diffusion $D_{i, e}$ and mobility $\mu_{i, e}$ coefficients for electrons and ions across and along the magnetic field. For relatively low neutral gas pressure such that $\lambda_{i} \gtrsim R, L$, the collissionless free-fall regime will be established along $B$ and for relatively strong magnetic fields such that ion larmor radius $\rho_{i} \ll \lambda_{i}$ the cross-field transport will be significantly suppressed. In this work we are not aiming to consider all transport regimes which could generally be realised in a magnetized plasma column. We focus instead only on those regimes which present most practical interest for particular conditions of magnetized helicon discharge plasmas. Readers interested in a broader understanding of different transport mechanisms are referred to several extensive review on this topic ${ }^{2-4}$. In the low-temperature helicon plasmas typically used in the laboratory experiments and industry the neutral gas pressure $p$ varies in the range from $0.1-1 \mathrm{~Pa}$, magnetic field $B$ in the range from $1-100 m T$, cylinder radius $R$ between $3-20 \mathrm{~cm}$. The typically achieved $\lambda_{i} \sim 7 \mathrm{~mm}$, $\rho_{i} \sim 3 \mathrm{~mm}$, electron Larmour radius $\rho_{e} \sim 0.1 \mathrm{~mm}$. Also, ions are typically only weakly magnetized, i.e. the ion-neutrals collision frequency $\nu_{i}$ is comparable or

a) Electronic mail: ilys@ipp.mpg.de higher than the ion gyrofrequency $\nu_{i} \sim f_{c i}$. For instance at IShTAR typically $\nu_{i} \sim 58 \mathrm{kHz}$ and $f_{c i} \sim 37 \mathrm{kHz}$. At these conditions the characteristic radial step size in the random walk ansatz (which is either $\lambda_{i}, \rho_{i}$ or $\rho_{e}$ ) is usually $\lesssim R, L$ and cross-field particle transport will be governed by collisions or turbulence rather than by collissionless free-fall. The question here is which collisional transport mechanisms play the dominant role in the cross-field transport. Meanwhile, which relative contribution of the perpendicular and parallel transports to the overall particle loss. The purpose of this work is to compare and demostrate the relative contribution of different transport mechanisms as well as the role of parallel and cross-field transports on the example of helicon plasma discharge at the IShTAR experiment.

\section{DESCRIPTION OF THE EXPERIMENTAL SETUP}

All measurements presented in this work have been done in the plasma source module of the IShTAR test facility. The plasma source module is composed of a lefthanded half turn helical antenna wrapped around the quartz vacuum vessel $(R=0.2 \mathrm{~m}$ and $L=1 \mathrm{~m}$ ) and permeated by an approximately uniform magnetic field. The strength of magnetic field $B$ can be set in the range from $0-64 \mathrm{mT}$. The antenna is $64 \mathrm{~cm}$ long and $44 \mathrm{~cm}$ in diameter. It launches fast and slow wave components and has such a helicity that it launches $m=+1$ helicon waves anti-parallel to the $B$ direction and $m=-1$ waves parallel to $B$. The measurements have been done for different neutral argon $A r$ gas pressures in the range $0.8-1.4 \times 10^{-3}$ mbar. The experiment is equipped with a positioning system enabling a one-dimensional scan in the radial direction with a spatial precision of $\approx 2 \mathrm{~mm}$. All results presented in this work has been obtained using single Langmuir probe measurements. The probe has been installed on the positioning system using an additional axial shaft to position the probe at a required axial location inside the plasma source. The typically achieved plasma density $n$ in the plasma source lies in 
the range of $n \sim 410^{17} \mathrm{~m}^{-3}$ and typical electron temperature $T_{e} \sim 5 \mathrm{eV}$. The detailed description of the IShTAR test facility can be found in Ref. ${ }^{1}$.

\section{DIFFERENT MECHANISMS OF PARTICLE TRANSPORT}

In this section several transport mechanisms will be considered wich could hypothetically contribute to the cross-field plasma transport. First, the general concept of a particle transport without a magnetic field will be considered. The particle transport can be either caused by particle collisions or it can collisionless. The collisional transport for specie $\alpha=e, i$ is expressed in the following form:

$$
\Gamma_{\alpha}= \pm \mu_{\alpha} n E-D_{\alpha} \nabla n
$$

Here index $\alpha$ corresponds to electrons $e$ or ions $i ; \Gamma_{\alpha}$ is the flux of particles; $\mu_{\alpha}$ and $D_{\alpha}$ are mobility and diffusion coefficients; $n$ is the plasma density; $E$ is the electric field. The diffusion and mobility coefficients for electrons and ions are defined by

$$
\begin{aligned}
D_{i} & =\frac{k_{B} T_{i}}{\nu_{i}} & \mu_{i} & =\frac{e}{M \nu_{i}} \\
D_{e} & =\frac{k_{B} T_{e}}{\nu_{e}} & \mu_{e} & =\frac{e}{m_{e} \nu_{e}},
\end{aligned}
$$

where $\nu_{i}$ and $\nu_{e}$ designate the ion and electron total collision frequency. In practice, for the typical helicon discharge conditions the ion-neutral collisions are predominant for ions $\nu_{i} \approx \nu_{i a}$. For electrons both electron-neutral and electron-ion collisions should be taken into account. The collisional ambipolar transport in the chamber with a dielectric wall requires that

$$
\Gamma_{i}=\Gamma_{e}
$$

This is the condition for the formation of the ambipolar electric field

$$
E_{a}=\frac{D_{i}-D_{e}}{\mu_{i}+\mu_{e}} \frac{\nabla n}{n} .
$$

The fact that $E_{a} \propto \nabla n$ allows us to combine the real diffusive motion under $\nabla n$ and a mobility drift under the action of the electric field $E$ introducing the ambipolar diffusion

$$
\Gamma=-D_{a} \nabla n
$$

with the ambipolar diffusion coefficient $D_{a}$ expressed as

$$
D_{a}=\frac{\mu_{i} D_{e}+\mu_{e} D_{i}}{\mu_{i}+\mu_{e}} .
$$

One should note, however, that the ambipolar diffusion is not a free diffusion in the general sense but instead just a convenient form to combine and express both mobility and diffusion dominated fluxes in a more concise form. This however is not always possible. For instance, if the cross-field radial transport is governed by other mechanisms which either do not form a radial electric field or this electric field is not proportional to $\nabla n$. In this case to evaluate the collisional radial particle transport the total momentum equations for electrons and ions should be considered. Exact two-dimensional solutions to these two coupled nonlinear equations is pretty complex.

An analysis of the particle diffusion in magnetic field requires a detailed consideration on the formation of space charge and currents flowing in plasma. The ambipolarity condition in the magnetized plasma takes sometimes a more relaxed form requiring only that overall wall-integrated electron and ion transports be equal. This occurs for example in the chamber with the conducting wall when ions undergo primarily cross-field transport and electrons diffuse predominantly along magnetic field, so that both species move in a mutually perpendicular direction.

Several mechanisms can hypotetically contribute to the cross-field particle losses in the typical helicon discharge tube. The flux $\Gamma_{a m b}$

$$
\Gamma_{a m b}=D_{\perp a} \nabla n
$$

corresponds to transport determined by the crossfield ambipolar diffusion coefficient in the magnetized plasma $^{4,5}$ in a chamber with dielectric walls

$$
D_{\perp a}=\frac{\mu_{\perp i} D_{\perp e}+\mu_{\perp e} D_{\perp i}}{\mu_{\perp i}+\mu_{\perp e}},
$$

where the subscript $\perp$ designates the cross-field coefficient. Practically this diffusion coefficient is entirely applicable only to the chamber where both side and end walls are dielectric so that no return currents can flow through the plasma. Dielectric walls break the current path not allowing to complete the circuit. The collisional radial transport in the plasma completely confined in the conducting wall is driven by the so called Simon diffusion

$$
\Gamma_{\text {Simon }}=D_{\perp S} \nabla n
$$

where $D_{\perp S}$ is given by ${ }^{4,5}$

$$
D_{\perp S}=\frac{\mu_{i} D_{\perp e}+\mu_{e} D_{\perp i}}{\mu_{i}+\mu_{e}},
$$

The real character of a collisional diffusion in a chamber with dielectric side wall and conducting end plates (as in most of helicon discharges) is more complex and is some sort of mixture of $\Gamma_{\text {Simon }}$ and $\Gamma_{a m b}$. The diffusion in the main plasma volume most likely is driven by the Simon mechanism since all flux tubes are short circuited through the end plates so that the current between any neighboring flux tubes can flow. The diffusion right at the plasma boundary will be still ambipolar since no current can flow from the side wall to the end plates. If the side wall of the chamber is inclined to the direction of $B$, the diffusion even in the chamber with the dielectric wall in not ambipolar, i.e. is not driven only by $D_{\perp a}$ and occurs much faster. Nevertheless, even if we do not know which of both collisional diffusion mechanisms play the dominant role and even if we do not know how precisely 
the side wall is aligned with the direction of the magnetic field, we always can say that the collisional transport $\Gamma_{\text {coll }}$ lies between

$$
\min \left\{\Gamma_{\text {Simon }}, \Gamma_{\text {amb }}\right\}<\Gamma_{\text {coll }}<\max \left\{\Gamma_{\text {Simon }}, \Gamma_{\text {amb }}\right\}
$$

An extended review on the collisional diffusion is given in Ref. ${ }^{2}$.

The existence of a radial electric field $E$, much stronger than the ambipolar one, indicates that there is a substantial ion acceleration potential $E \cdot R \gg T_{i}$. In this case the perpendicular ion transport is governed by the ion mobility term rather than by the diffusion and in this case the preceeding discussion about the ambipolar diffusion of any kind is unapplicable. If such an electric field exist then the transport is determined by the ion mobility

$$
\Gamma_{\mu}=n \mu_{i} E_{r}
$$

It will be shown later in this work that the ion mobility dominated transport could also play a substantial role at the plasma boundary since the corresponding electric field there is not always negligible. Moreover, experiment and modelling on different transport mechanisms in Ref. ${ }^{3}$ suggest the decisive role of the ion mobility dominated transport.

Except of different collisional transport mechanism, there is also the turbulent diffusion driven by plasma instabilities. This mechanism is not driven by collisions and can exist even in near collisionless plasma. The corresponding turbulent transport

$$
\Gamma_{t u r b}=D_{t u r b} \nabla n
$$

occurs with the diffusion coefficient

$$
D_{\text {turb }}=A \frac{k_{B} T_{e}}{B},
$$

where $A$ is the coefficient of order unity. The coefficient $D_{\text {turb }}$ with $A=\frac{1}{16}$ is often called the Bohm diffusion coefficient and has been introduced by Bohm from the intuitive considerations. In our estimations of the turbulent transport we assume $A=1$.

From the experimental point of view the particle flux density to the wall $\Gamma$ can be easily estimated using Langmuir probe technique measuring the plasma density $n$ and electron temperature $T_{e}$ right at the plasma boundary i.e. at the sheath edge (the interface between sheath and pre-sheath)

$$
\Gamma=n_{s} u_{B}
$$

with $n_{s}$ corresponding to the sheath edge density and $u_{B}=\frac{k_{B} T_{e}}{m_{e}}$ corresponding to the Bohm velocity, where $m_{e}$ is the electron mass and $k_{B}$ is the Boltzmann constant. Two unobvious facts simplify an experimental estimation of $\Gamma$ :

- First, the plasma density at the plasma boundary is never equals to zero. Even though some simplified solution of the diffusion equation use zero
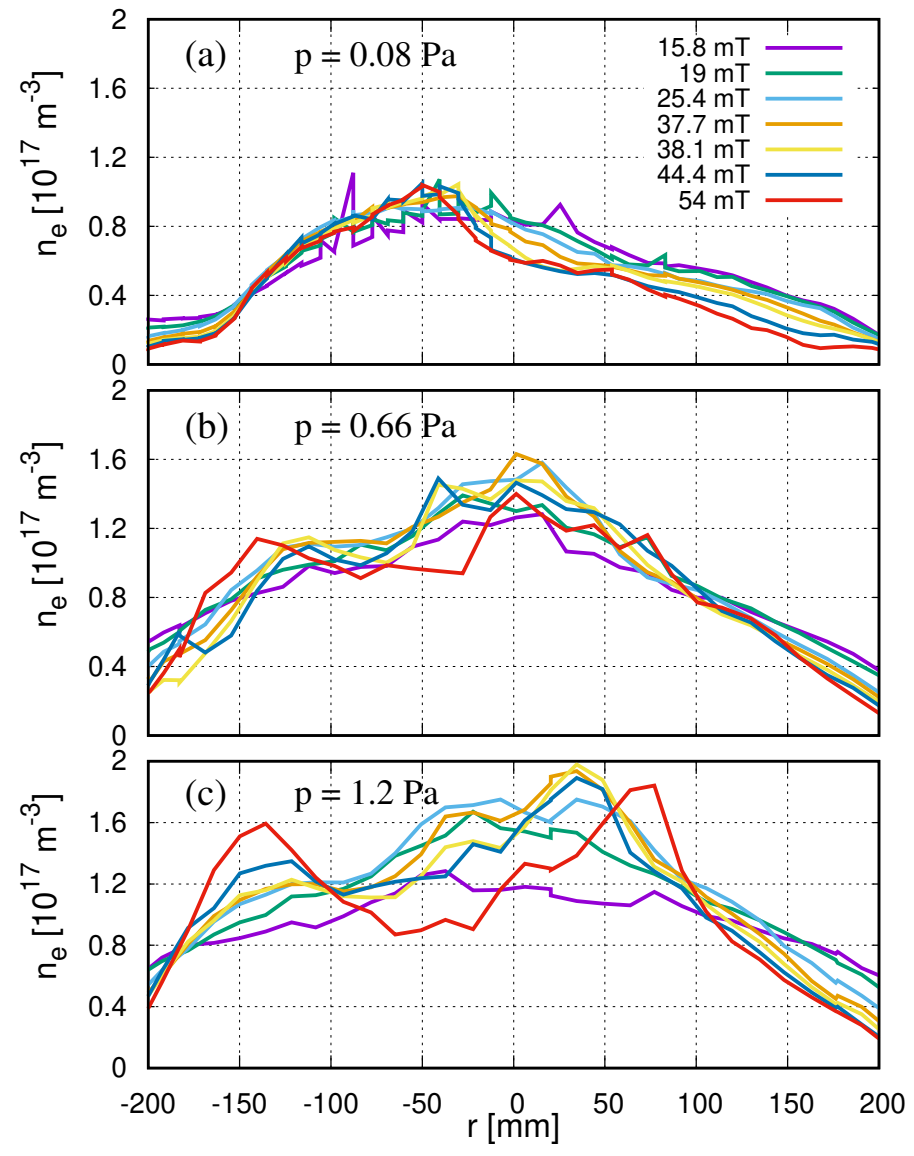

FIG. 1. The radial distribution of the plasma density measured for different values of the axial magnetic field. Results for three different neutral gas pressures are presented: (a) for $p=0.08 \mathrm{~Pa}$, (b) for $p=6.6 \mathrm{~Pa}$ and (c) for $p=1.2 \mathrm{~Pa}$. Each figure compares profiles measured for different strengths of an axial magnetic field in the range from $15-55 \mathrm{mT}$.

density boundary condition at the wall, this is just a first approximation which simplifies the theoretical consideration. The results on the modelling of the whole set of ion and electron momentum and continuity equations in Ref. ${ }^{6,7}$ show that density at the plasma boundary always converge to a certain finite value, which could be lower than the central plasma density only by several times. The density at the plasma boundary can be easily measured due to the fact that the sheath thickness is in the order of several tens of $\mu \mathrm{m}$ and much smaller than the typical diameter of the probe pin (in our case $0.8 \mathrm{~mm}$ ). Practically when the probe pin is positioned at the plasma boundary right up to the wall surface, the major part of the probe collection area will be still in the presheath and these measurements give a direct estimation of $n_{s}$.

- Second, for the typical helicon discharge plasmas the plasma particle velocity at the plasma boundary approaches $u_{B}$, even in the presence of the axial magnetic field. The modelling results in Ref. ${ }^{4,6,7}$ demonstrate that the Bohm criterion is still satisfied also for the motion across $B$. The electric force on the electrons dominates the magnetic Lorentz 

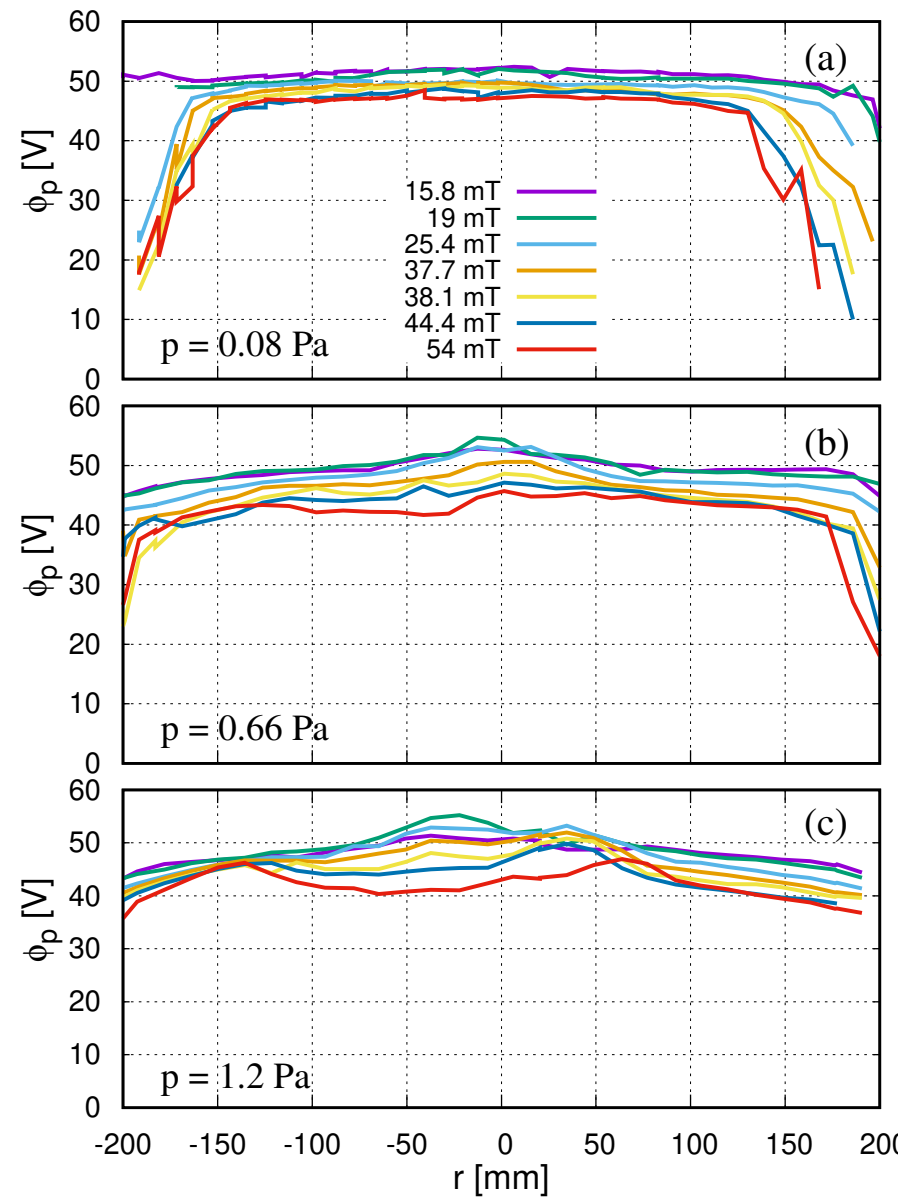

FIG. 2. The radial distribution of the plasma potential measured for different values of the axial magnetic field. Results for three different neutral gas pressures are presented: (a) for $p=0.08 P a$, (b) for $p=6.6 P a$ and (c) for $p=1.2 P a$. Each figure compares profiles measured for different strengths of an axial magnetic field in the range from $15-55 \mathrm{mT}$.

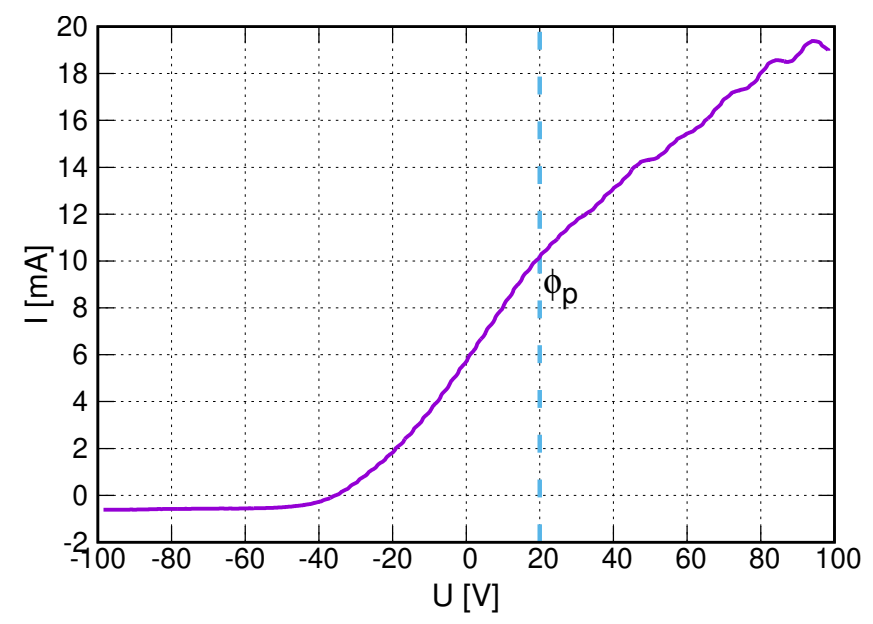

FIG. 3. The typical probe I-U characteristics measured at the plasma edge at the radial position of $r=-200 \mathrm{~mm}$ for the neutral gas pressure $p=0.08 \mathrm{~Pa}$ and axial magnetic field $B=54 \mathrm{mT}$, when the observed edge radial electric field is highest. The blue dashed line indicates the position of the plasma potential. The characteristics does not show any artefacts leading to an ambiguous estimation of $\phi_{p}$.

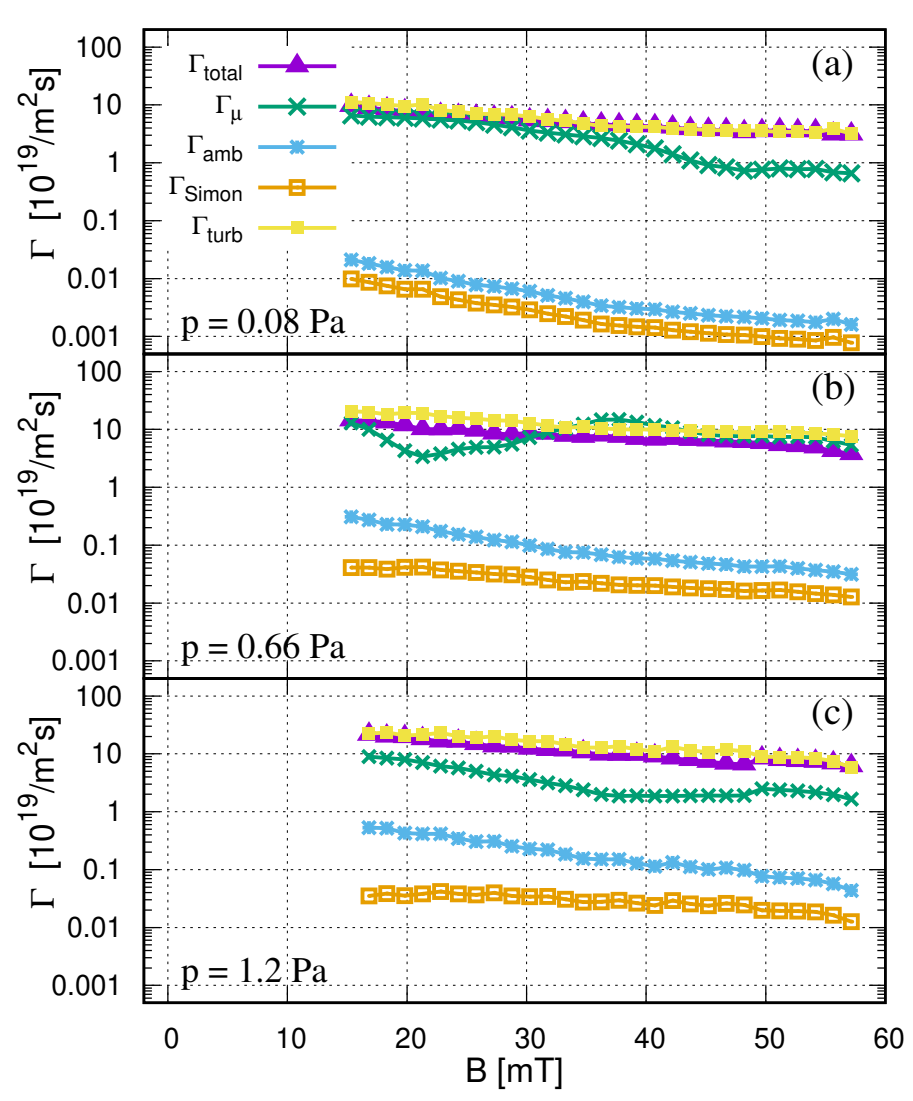

FIG. 4. Estimation of the contribution of different transport mechanisms to the total radial particle flux density in the magnetized plasma discharge in $A r$. Results for three different neutral gas pressures are presented: (a) for $p=0.08 P a$, (b) for $p=6.6 \mathrm{~Pa}$ and (c) for $p=1.2 \mathrm{~Pa}$. Results are presented as a function of the axial magnetic field strength. $\Gamma_{\text {total }}$ corresponds to the total particle flux density. $\Gamma_{\mu}$ corresponds to the flux density determined by the ion mobility. $\Gamma_{a m b}$ represents the flux due to the ambipolar cross-field diffusion. $\Gamma_{\text {Simon }}$ represents the cross-field diffusion governed by the Simon mechanism (chamber with the conducting walls). $\Gamma_{\text {turb }}$ is the turbulent cross-field diffusion.

force in the sheath region. Also the experimental indication of enhanced outward ion velocity which has been cited in Ref. ${ }^{8}$. The application of the laser induced fluorescence (LIF) technique shows the radial ion velocities at the plasma boundary close to $u_{B}$, implying an acceleration of ions toward the wall similar to that in the absence of a magnetic field. Apparently, one could not generalize this thesis to any arbitrary high magnetic field, i.e. it is applicable only up to a certain magnetic field strength. However, the above mentioned references suggest an applicability for typical helicon discharge plasmas.

\section{MEASUREMENTS}

The results presented in this work are based on a systematic measurements of $n$ and $\phi_{p}$ radial profiles for different pressure and magnetic field values. Results in the 
Fig.1 present $n$ profile for $p=0.08 P a$ (a), 0.66 $P a$ (b), $1.2 \mathrm{~Pa}$ (c). The radial profiles for different $B$ in the range from $15-54 m T$ are compared. The scan has been performed throughout the entire radial range so that the left-most and right-most positions correspond to the density at the chamber wall. The density measurements at these points correspond to $n_{s}$ and is directly related to the radial particle transport. Even though the magnetic field does not play a big role on the central plasma density, increase in $B$ significantly and consistently reduces the edge density. This is qualitatively in agreement with the general concept of reduction of radial losses with the magnetic field.

Along with the diffusion, the mobility dominant transport described in Eq.13 could also contribute to the particle losses when the radial electric field is sufficiently high. The radial distribution of the plasma potential is presented in Fig.2. Results have been measured in the same series of experiments as the one in Fig.1 and therefore are presented in the same manner. Results for different magnetic field values and neutral gas pressure $p$ of $0.08 \mathrm{~Pa}$ (figure (a)), $0.66 \mathrm{~Pa}$ (figure (b)) and 1.2 Pa (figure (c)) are presented. For low pressure value $p=0.08 \mathrm{~Pa}$ in Fig. (a) $\phi_{p}$ profile is pretty flat in the bulk plasma in the entire presented range of magnetic field strengths. This is however not the case at the plasma edge where $\phi_{p}$ experiences a sharp fall, indicating the presence of a strong radial electric field in the range of $\sim 1000 \mathrm{~V} / \mathrm{m}$. This electric field does not have an ambipolar nature. Simple estimation of the ambipolar electric field

$$
E_{\perp a}=\frac{D_{\perp i}-D_{\perp e}}{\mu_{\perp i}+\mu_{\perp e}} \frac{\nabla n}{n}
$$

using the plasma density radial profiles measured at the plasma boundary (taken from the Fig.1) gives the value in the order of $4 \frac{V}{m}$, much lower than the observed value. Also, if we take into account that end plates are conducting and therefore equipotential, the radial electric field is determined by the potential drop between the wall and the bulk plasma ${ }^{4}$

$$
\Phi_{w}=-T_{e} \ln \left(\frac{M}{2 \pi m_{e}}\right)+\frac{T_{e}}{2},
$$

where $M$ designate an ion mass. For $A r$ ions it gives $\Phi_{w}=-5.2 T_{e}$ and the corresponding radial electric field

$$
E_{\perp c o n d}=5.2 \nabla T_{e} .
$$

Practically $T_{e}$ profile is pretty flat due to a high electron thermal conductivity and the difference in $T_{e}$ between the plasma centre and boundary constitute not more than $1 \mathrm{~V}$ producing $E_{\perp \text { cond }} \sim \frac{5.2 \cdot 1}{0.2}=26 \frac{\mathrm{V}}{\mathrm{m}}$. This is still way smaller than the observed $\sim 1000 \frac{\mathrm{V}}{\mathrm{m}}$ at the edge.

The presence of a diamagnetic current flowing in the poloidal direction due to $\nabla p$ could also contribute to the radial electric field

$$
E_{\perp \text { diam }}=\frac{\nabla p}{e n} .
$$

This contribution, however, $E_{\perp \text { diam }} \sim \frac{5 \mathrm{eV}}{0.2 \mathrm{~m}}=25 \frac{\mathrm{V}}{\mathrm{m}}$, is also way to small.

The layer of a strong radial electric field is also observed for an intermediate pressure $p=0.66 \mathrm{~Pa}$ in Fig.(b), but it is already invisible for $p=1.2 \mathrm{~Pa}$ in Fig.(c) either because the electric field becomes lower or because the layer becomes thinner. Thus, the origin of the observed strong radial electric field at the edge is not clear.

Hypothetically it could be that the observed enhanced radial electric field at the plasma edge in reality caused by some kind of distortion of the measured probe current-voltage (I-U) characteristics. Such a distortion could be caused, for instance, by the so-called "current overshoot" 9 or sheath rectification due to an oscillating plasma potential in the RF environment ${ }^{10}$ (even though the RF compensated probes ${ }^{11}$ are used to measure probe data in the RF environment). Therefore, the typical probe characteristics is shown in Fig.3 for reference in order to dispel doubts that the observed electric field arises from the misinterpretation of the observed IU curves. This characteristic has been measured at discharge conditions when the observed edge radial electric field is highest (and supposedly also highest distortion), i.e. at low neutral gas pressure $p=0.08 \mathrm{~Pa}$, high magnetic field $B=54 \mathrm{mT}$ and directly at the plasma boundary $r=-190 \mathrm{~mm}$, as visible in Fig.2(a). The blue dashed color line indicates the position of the plasma potential $\phi_{p}$ measured at the place where the slope of the probe characteristic changes the value. The observed characteristic does not show any obvious artefacts allowing to doubt that it is correct.

The role of the observed enhanced radial electric field on the particle transport will be analysed later in this work.

\section{EXPERIMENTAL ANALYSIS OF DIFFERENT TRANSPORT MECHANISMS}

An experimental estimation of cross-field particle transport to the wall has been performed systematically for a neutral gas pressures $p$ from $0.08-1.2 P a$ and an external magnetic field $B$ in the range from $14-60 \mathrm{mT}$. These results are presented Fig. 4. Here we compare the relative contribution of different transport mechanisms to the total particle transport to the wall $\Gamma_{\text {total }}$, estimated from Eq.16. The contributions of $\Gamma_{\mu}, \Gamma_{a m b}, \Gamma_{\text {Simon }}$ and $\Gamma_{\text {turb }}$ are estimated from Eqs. 13, 8, 10 and 14, respectively.

These measurements correspond to the radial position right in the vicinity of the plasma boundary $r=200 \mathrm{~mm}$. Therefore all corresponding plasma parameters including the radial electric field and plasma density gradients have also been taken in the boundary region. Results for three neutral gas pressures $p$ of $0.08 \mathrm{~Pa}$ (figure (a)), 0.66 Pa (figure (b)) and 1.2 Pa (figure (c)) are presented.

Results in Fig.4(a) show that among different transport mechanisms, the main contribution to $\Gamma_{\text {total }}$ is provided by $\Gamma_{\text {turb }}$. Contributions provided by collisional diffusions $\Gamma_{\text {Simon }}$ and $\Gamma_{a m b}$ are approximately three orders 
of magnitude below $\Gamma_{\text {total }}$. Most likely the collisional diffusion dominated transport in the chamber with conducting end plates (like the one at IShTAR) is between $\Gamma_{\text {Simon }}$ and $\Gamma_{a m b}$, as has been stated in Eq.12. So the collisional diffusion does not play any significant role in the outward transport for this pressure. The ion mobility dominated transport $\Gamma_{\mu}$, however, does play a substantial role. This is because the radial electric field, relatively modest in the bulk plasma, approaches significant values at the boundary.

Looking at results for higher pressures in Fig.4(b) and (c) one can notice that even though the diffusive transport $\Gamma_{\text {Simon }}$ and $\Gamma_{a m b}$ becomes way higher due to an increase in the ion-atom collision rates $\nu_{i a}$ from $9 \mathrm{kHz}$ to $115 \mathrm{kHz}$, it still remains way to small in order to provide a significant contribution to $\Gamma_{\text {total }}$. The mobility dominated transport $\Gamma_{\mu}$, playing relatively important role for an intermediate pressure in Fig.4(b), plays gradually a less important role for higher pressures in Fig.4(c). It turns out that $\Gamma_{\text {turb }}$ is the only transport mechanism which always show consistent agreement with $\Gamma_{\text {total }}$, both quantitative and qualitative and in a wide range of discharge parameters. One can always argue about a quantitative consistency since the choise of the coefficient $A$ in Eq.15 is somewhat artificial. The qualitative consistency, however, looks more convincing because of a remarkable similarity in the dependence of $\Gamma_{t u r b}$ and $\Gamma_{\text {total }}$ on $B$, i.e. similar slope.

The obvious question often arises in a design of a new plasma source: which transport, parallel or perpendicular determine the overall particle losses? The plasma lifetime (or confinement time) $\tau$ will be introduced in order to answer on this question. It can be estimated as follows:

$$
\tau=\frac{\bar{n} V}{\Gamma A}
$$

where $\bar{n}$ designate the volume averaged density, $V$ is the entire plasma volume so that the product $\bar{n} V$ is the total number of particles in the volume. $\Gamma$ is the outward particle flux density and $A$ is the surface area which plays the role of the particle sink. In the magnetized plasma one can distinguish between the parallel and perpendicular confinement times, depending on the direction of $\Gamma$ with respect to $B$

$$
\tau_{\|}=\frac{\bar{n} V}{\Gamma_{\|} A_{\text {end }}}
$$

and

$$
\tau_{\perp}=\frac{\bar{n} V}{\Gamma_{\perp} A_{\text {side }}}
$$

where $A_{\text {end }}=2 \pi R^{2}$ and $A_{\text {side }}=2 \pi R L$ designate the surface area of the end plates and the side wall. The plasma lifetime $\tau$ is of the order of the smallest between $\tau_{\|}$and $\tau_{\perp}$. The ratio

$$
\frac{\tau_{\|}}{\tau_{\perp}}=\frac{\Gamma_{\perp} A_{\text {side }}}{\Gamma_{\|} A_{\text {end }}}
$$

shows the relative contribution of parallel and perpendicular transports. Note that apart from the flux density it includes also the dimensions of the vessel. The lifetime will be primarily governed by the parallel transport if $\frac{\tau_{\|}}{\tau_{\perp}} \ll 1$ and cross-field transport if $\frac{\tau_{\|}}{\tau_{\perp}} \gg 1$. The $\frac{\Gamma_{\perp}}{\Gamma_{\|}}$ can be estimated as follows

$$
\frac{\Gamma_{\perp}}{\Gamma_{\|}} \approx \frac{D_{\perp} L}{D_{\|} R},
$$

where $D_{\perp}$ and $D_{\|}$designate the perpendicular and parallel diffusion coefficients; $L$ and $R$ designate the length and radius of the plasma source chamber. The parallel transport is governed by the ambipolar diffusion coefficient in the absence of the magnetic field $D_{\|}=D_{a}$ given in Eq.7. The perpendicular transport is governed mostly by the turbulent diffusion $D_{\text {turb }}$, as we have seen from Fig.4. The Eq.25 can be rewritten in the following form

$$
\frac{\Gamma_{\perp}}{\Gamma_{\|}}=\frac{D_{\text {turb }} L}{D_{a} R},
$$

For discharge parameters typically used at IShTAR $B \approx$ $30 \mathrm{mT}$, a neutral gas pressure $p \propto 0.6 P a$, the corresponding coefficients are $D_{a} \approx 200 \frac{\mathrm{m}^{2}}{\mathrm{~s}}$ and $D_{\text {turb }} \approx$ $160 \frac{m^{2}}{s}$. The corresponding ratio $\frac{\tau_{\|}}{\tau_{\perp}} \approx 7$, which indicate a predominant role of the cross-field particle diffusion in the overall particle transport. Note, however, that $D_{a}$ has a strong dependence on the neutral gas pressure $p$ since it depends primarily on ion-atom cillisions $\propto \frac{1}{\nu_{i a}}$. The turbulent diffusion coefficient $D_{\text {turb }}$, in turn, does not depend on $p$, at least in the first approximation Therefore, one can expect a bigger role of the parallel transport for lower $p$. This is indeed the case since for $p=0.1 \mathrm{~Pa}$ and for the same $B \approx 30 \mathrm{mT}$ we get $\frac{\tau_{\|}}{\tau_{\perp}} \approx 1$ so that both parallel and perpendicular losses are comparable.

The last discussion has an important practical consequences on the design of a helicon plasma source. There is always a demand to design a more powerfull helicon plasma source, i.e. able to produce a more dense plasma. However the RF power which can be applied to a single helicon antenna is limited since parasitic RF arcing emerges between different antenna elements when RF power approaches a certain threshold level. In order to avoid this, the applied RF power is sometimes distributed between several helicon antennae arranged parallel along the vacuum tube. This approach extends the lengths of the tube, inevitably rising the fraction of particles escaped radially. Apparently, the efficiency of this approach will be very low if $\frac{\tau_{\|}}{\tau_{\perp}} \gg 1$.

\section{CONCLUSION}

The turbulent transport plays the dominant role in the cross-field particle losses exceeding the ambipolar 
diffusion of any kind $\left(\Gamma_{a m b}\right.$ or $\left.\Gamma_{\text {Simon }}\right)$ by $2-3$ orders of magnitude. $\Gamma_{\text {turb }}$ consistently show a good qualitative and quantitative agreement with the total losses for all presented pressures and magnetic fields. An existence of a strong radial electric field at the plasma boundary, determining the ion mobility dominant transport, could also significantly contribute to the radial losses. A comparison between the total parallel and perpendicular losses shows that the perpendicular ones can be even larger or at least comparable to the parallel ones. This has an important practical meaning: multiplying the number of antenna arrangen parallel along a vacuum tube trying to distribute an applied RF power could be much less effective than if the entire power is applied to a single helical antenna with a short vacuum vessel.

\section{ACKNOWLEDGMENTS}

This work has been carried out within the framework of the EUROfusion Consortium and has received funding from the Euratom research and training programme 2014-2018 and 2019-2020 under grant agreement No 633053. The views and opinions expressed herein do not necessarily reflect those of the European Commission.

${ }^{1}$ I. Shesterikov et al., Review of Scientific Instruments (in press 2019b).

${ }^{2}$ A. P. Zhilinsky and L. D. Tsendin, Soviet Physics Uspekhi 23, 331 (1980).

${ }^{3}$ F. Vidal et al., IEEE Transactions on Plasma Sciences 27, 727 (1999).

${ }^{4}$ M. A. Lieberman and A. J. Lichtenberg, Principles of Plasma Discharges and Materials Processing, 2nd ed. (WILEY, 2005).

${ }^{5}$ V. E. Golant, A. P. Zhilinsky, I. E. Sakharov, and S. C. Brown, Fundamentals of Plasma Physics (New-York, WILEY, 1980).

${ }^{6}$ J. E. Allen and J. T. Holgate, Nuclear Materials and Energy 12, 999 (2017).

${ }^{7}$ D. Curelli and F. F. Chen, Plasma Source Sci. Technol. 23, 064001 (2014).

${ }^{8}$ M. A. Lieberman and R. A. Gottscho, "Design of high-density plasma sources for materials processing," (Academic Press, San Diego, California, 1994) Section VIII, in "Physics of Thin Films" V.18.

${ }^{9}$ R. L. Stenzel and J. M. Urrutia, Phys. Plasmas 4, 26 (1997).

${ }^{10} \mathrm{~N}$. Hershkowitz, "Plasma diagnostics. discharge parameters and chemistry," (Academic Press, San Diego, California, 1980).

${ }^{11}$ I. D. Sudit and F. F. Chen, Plasma Sources Science and Technology 3, 162 (1994). 